\title{
Whole Body Personnel Monitoring via Ionization Detection
} \section{J.E. Koster, J.A. Bounds, P.L. Kerr, P.A. Steadman, and C.R. Whitlegy
Los Alamos National Laboratory, Los Alamos, NM 87545$$
\text { CONF-971147-- }
$$ \\ II. MONITOR CONSTRUCTION

\begin{abstract}
A project between Fernald EMP and LANL is to field a monitor for the detection of alpha-emitting contamination on a human body. Traditional personnel monitoring for alpha emitters involves either frisking with a probe or pressing against large detectors in order to overcome the short range of alpha particles. These methods have a low alpha collection efficiency, and can miss contamination on less accessible surfaces. We have investigated the sensitivity and practicality of measuring the entire subject simultaneously using the technique of ionization monitoring. The goal is to create a booth that personnel step into quickly during egress from radiological facilities. The detection technique relies on a breeze of air passing over the subject. Alpha emission produces copious ions in the ambient air which are transported by the air current to an ion collector, resulting in a small electrical current proportional to the amount of contamination. Results indicate a conservative sensitivity of 3000 disintegrations per minute localized to one of five areas of the body in a measurement lasting less than 2 minutes.
\end{abstract}

\section{INTRODUCTION}

Personnel leaving DOE facilities and sites where radiological materials are present are subject to monitoring in order to detect any contamination and prevent its spread. Often, the material present includes actinides (such as thorium, uranium or plutonium), which are prolific alphaparticle emitters and in some cases difficult to detect via other radiation. The present monitoring of personnel for actinides can involve two techniques. Personnel are frisked with a small alpha probe, a time-consuming and labor intensive method which relies upon the consistency and care of the personnel doing the frisking. Personnel may also use fulllength stationary alpha monitors which require pressing various sides of the body in turn against the sensitive surfaces of the alpha monitor.

This paper presents the use of ionization monitoring to detect the presence of actinides on the body in a sensitive, simultaneous measurement. Ionization monitoring measures the amount of ionized air created by alpha particles emitted by decaying actinides. The air ions are transported to ion collection plates by air currents. Contamination which exists in less accessible areas (for example, between the fingers) is more likely to be detected via ionization monitoring as long as some air current is nearby. This is because the ions can be carried much farther in an airstream than the range of the alpha particles alone. This increase in detection range has been termed Long-Range Alpha Detection (LRAD).

\section{A. Principle of Operation}

Radioisotopes can be detected via the ionization produced in air by collisions between emitted particles and air molecules. In a small (liters or less) volume of air, alpha particles are particularly well-suited to such ionization monitoring because of their short range and high rate of ionization. The mean energy expended by an alpha particle in a collision (with an air molecule) resulting in an ion pair is approximately $35 \mathrm{eV}$. Thus, the typical alpha particle emitted by an actinide nucleus, having $\sim 5 \mathrm{MeV}$ energy, will produce about 140,000 ion pairs. The electron liberated in an ionization quickly attaches to a neutral air molecule creating equal numbers of positive and negative ions. The ions are carried in air currents to an electrode, where a sensitive electrometer measures the resulting current from the half of the ions with the appropriate polarity.

Detecting 140,000 ions rather than a single alpha particle directly provides several benefits. The relatively slow recombination rate of ions in the diffuse ion cloud allows them to be collected from a substantial distance - as far as several meters - compared with the range of a typical alpha particle of a few centimeters in air. This advantage admits some stand-off distance of the (ion) detector from the body. A second advantage is that quicker monitoring may be possible. The presence of nuclear material or contamination usually produces many more ions than effectively result from background events (cosmic rays and terrestrial external radiation entering the detector, and radon gas) and therefore the signal due to the contamination can be observed above background levels quickly. A third advantage is the good sensitivity that may be obtained (even with a quick measurement). In a traditional detector, either the alpha particle reaches the monitor and is detected or it does not. However, only some fraction of the 140,000 ion pairs must reach the LRAD-based ionization monitor in order to register an alpha event. Thus, some ion losses can be tolerated in the system while retaining greater sensitivity than a direct alpha particle detector.

The final advantage of ionization monitoring is the potential for a simultaneous measurement of the entire body. The ions move on air currents created by fans. The air can be drawn across all surfaces of the body and then into the ion collector. Traditional detection of the alpha particle within a monitor means that only the parts of the body within an inch of detector surface are monitored at that instant. Personnel performing a frisking must move the alpha probe 


\section{DISCLAIMER}

This report was prepared as an account of work sponsored by an agency of the United States Government. Neither the United States Government sor any agency thereof, nor any of their employees, makes any warranty, express or implied, or assumes any legal liability or responsibility for the accuracy, completeness, or usefulness of any information, apparatus, product, or process disclosed, or represents that its use would not infringe privately owned rights. Reference herein to any specific commercial product, process, or service by trade name, trademark, manufacturer, or otherwise does not necessarily constitute or imply its endorsement, recommendation, or favoring by the United States Government or any agency thereof. The views and opinions of authors expressed herein do not necessarily state or reflect those of the United States Government or.any agency thereof. 
collection plates also serve to isolate the electrical fields in the plate region. The electric field between plates is sensitive to objects and motion outside the ion collection region if enabled to extend there by the absence of such an electrical "cage". In addition, the guard on the downstream side of the plates may help reduce electrical noise from the fans.

The circuitry for biasing the plates is design to mitigate any shock hazard. The guard sheet between the monitoring chamber and the collection region also serves to keep personnel from falling into the plates.

Both the air filters and the guard sheets have an unfortunate consequence of providing resistance to the air flow. The air filter density was chosen to optimize the ion filtration and the air speed. The guard sheets' perforation sizes are chosen as large as possible while considering safety, electric field containment, and filter material support, as appropriate. Perforated metal was used initially but with approximately $50 \%$ surface area these sheets cut down the airspeed too much. Expanded metal is used to provide a much reduced surface area in the air stream.

The ion collection plates are installed in a stack of 21 . Plates alternate between grounded and biased voltage. Every two biased plates are electrically connected. This provides 5 sets of signal plates. Ion collection is thereby segmented vertically in order to investigate localization of any contamination on the body. The segments extend from 0-43, $43-85,85-128,128-171$, and $171-213 \mathrm{~cm}$ from the floor respectively.

An electrometer is installed on a signal plate for each pair of signal plates. The currents produced at the signal plates can be as small as $10^{-15}$ amperes ( 1 femtoamp). The electrometers convert these small currents to voltage signals ranging from millivolts to volts. The 5 voltage signals are passed outside the test booth to a scanning digital voltmeter which is connected in turn to a desktop computer for data acquisition, display, and storage. The electrometers have been designed by LANL and used in a variety of ionization monitors. A Keithley 199 and a Macintosh computer running LabView are used for the data acquisition system. Integrators connected to each electrometer provide a 5 second integration time for smoothing the signals. This integration in hardware is optional.

\section{RESULTS}

The key parameters describing the capability of the laboratory test booth are the measurement time, the minimum detection limits, and the sensitivity. The count time is relatively short given that the booth has not been refined. After opening the door to step into the booth, the signals recover in about 20 seconds. A 1 to 1.5 minute count time suffices for all measurements but the ones near background. Therefore total measurement time is about 2 minutes. This is similar to what a careful, thorough frisking of the entire body would take.

The minimum detectable level in this test booth is 3000 disintegrations per minute (dpm). This may be improved upon with a smaller chamber and higher airflow. The current test chamber is $91 \mathrm{~cm} \times 91 \mathrm{~cm}$ but a smaller albeit cramped chamber could be used. Airflow effects will be described below. The minimum detectable level can be a factor of 2 smaller in ideal cases of personnel and contamination positioning within the booth. In a real measurement, however, the location of contamination will be unknown a priori and so we use a conservative figure.

The sensitivity of the monitor is $0.05-0.07 \mathrm{mV} / \mathrm{dpm}$ in individual segments of the monitor and $0.03 \mathrm{mV} / \mathrm{dpm}$ for the average of the 5 voltage signals. This implies a reducod efficiency compared to other LRAD-based monitors. The larger volume of airflow is probably an important factor.

\section{A. Airflow}

It was expected that sensitivity (ion collection efficiency) would increase with airspeed up to a point where airflow became turbulent. Furthermore, for a given airspeed the ion collection signal will decrease as the contamination is moved away from the collection plate region. These effects are due to ion recombination as described above. The effects may be reduced here because recombination is dependent largely on ion collisions with surfaces and in the test booth the surfaces (walls) are comparatively distant from typical locations of contamination (except near the feet).

With regard to airspeeds, however, some of the test results are inconsistent with expectations. For example, the net signal for a contamination source located at the chest is plotted in Fig. 2 as a function of airspeed ("fan frequency") and source distance ("position") from the collection plates guard sheet. Only the second and third pairs of plates responded appreciably to a source in this location (as expected - see the discussion on location sensitivity later). (Higher signals are more negative in voltage due to collection of negative ions.) However, the response of the second pair of plates (Fig. 2) is smallest for a source near the center of the booth, while the response is opposite (at least at higher airspeeds) in the third pair of plates. In addition, when the two signals are added the summed signal from a source at the center of the booth is lower than for a source at either extreme. That is, there is an unexpected ion loss for a source at the center of the booth. At the higher airflow, the response of the summed signal vs. distance is flattened out - yet the response increases with increasing separation of source from ion collector.

For some source positions a higher airflow has little effect on or even seems to reduce the signal. The airflow inside the booth can be expected to be turbulent - especially with a person inside. However, the airspeeds in these tests are fairly small in comparison to the cross-sectional area of the flow. It is not clear why we see these dramatic shifts of airflow from one set of plates to another or observe increased ion losses in some locations as the airspeed changes.

The response of the monitor to these parameters makes it difficult to establish an ion lifetime for this measurement geometry. The ion lifetime would enable us to calculate expected improvements in detection sensitivity with both higher airspeeds and smaller booth volumes. 
measurements with an unoccupied booth (comparing the two background points to the uncontaminated points in Fig. 3).

\section{E. Variability and Reproducibility}

Variability in the five signals is observed to be at worst $150 \mathrm{mV}$. Based on the sensitivity of the monitor, this corresponds to 2000 to $3000 \mathrm{dpm}$ in accord with the minimum detection level. However, short term reproducibility was normally good. For example, in contiguous sets of data the variation from one data average to the next was commensurate with the fluctuation within each data set.

Variations over time scales of hours are due to changes in natural background including primarily the radon concentration. The very large volume of the monitor makes it a sensitive radon detector [6]. Other background due to cosmic rays and background gamma radiation should be fairly constant and indeed was monitored at about $10 \mathrm{microrem} / \mathrm{hr}$.

\section{FUTURE DIRECTIONS}

The largest uncertainty in refining the design of the whole body monitor is the behavior of the air currents and their effect on ion collection. The data examples presented above clearly illustrate the issues that airflow is not uniform and ion collection does not vary in a simple manner across the volume of the monitoring chamber. This nonuniformity makes it difficult to predict the improvement in the minimum detection limit that might obtain with refinements.

Replacing the three large fans with many smaller ones may help make the airflow more even and predictable. Higher air blowing capacity may also be desirable.

The second refinement that can be done easily is to reduce the monitor volume. A smaller monitor will still accommodate most people. In fact, for the purposes of testing, a much smaller monitor should be built to contrast with the current booth. The refined monitor could be, for example, $61-76 \mathrm{~cm}$ on each side and $2 \mathrm{~m}$ high. The reduction in monitor volume means that both the linear airspeed will increase and the background will decrease.

The monitor must take background measurements continuously. A difference algorithm will be used when the booth is occupied to compare to the latest background measurement. This will mitigate the slow variation with ambient radon concentration. If the airflow itself and the ion behavior without airflow are better understood then it might be possible to quantify a correction for the reduction in background when the booth is occupied during a measurement.

The dependence upon body orientation can be handled in a couple of ways. The body could slowly rotate within the booth, perhaps on a rotating platform. More simple is the design of Fig. 4, in which air enters and exits the booth from two sides. The best measurement will probably be obtained

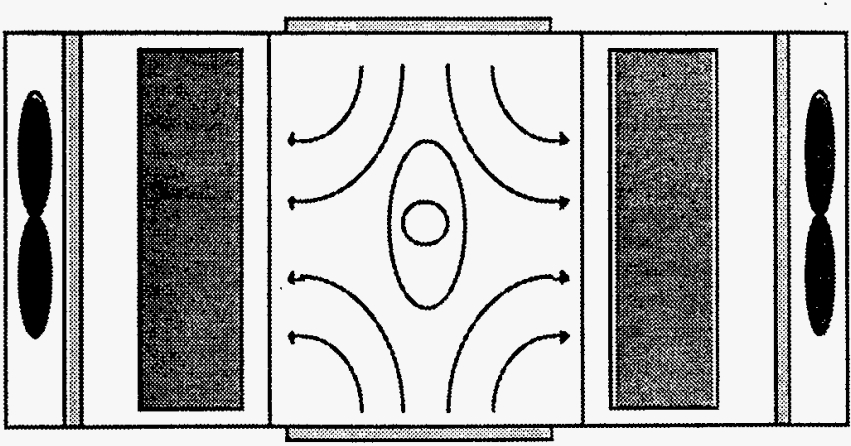

Fig. 4. Schematic of personnel monitoring in a booth with dual detectors. Air is drawn through filters on two sides of the chamber, passing around a potentially contaminated worker. Ions created by any contamination present are drawn with the air into either stack of electrically charged plates where a tiny current is produced and measured.

with the body oriented at 45 degrees (not as shown). This refinement could be extended with a 6 - or 8 -sided booth but the increase in mechanical complexity is probably not warranted.

\section{CONCLUSIONS}

The results of laboratory tests with personnel in a test booth show promise for the quick measurement of external contamination on almost all body surfaces. Minimum detection limits attainable upon further refinement may be competitive with limits from other monitoring techniques. A monitor of this type will permit better coverage of personnel for improved protection against the spread of contamination.

\section{REFERENCES}

[1] S.P. Rojas, et al., "Alpha characterization inside pipes using ion-transport technology," Proc. Waste Management '95, Folio Infobase CD-ROM.

[2] J.E. Koster, et al., "Real-time monitoring for alpha emitters in high-airflow environments," Proc. Nuclear and Hazardous Waste Management International Topical Meeting (SPECTRUM '96), Seattle, WA, August, 1996.

[3] R.W. Caress, et al., "LRAD-based airflow monitors," Los Alamos National Laboratory Internal Report, LA12742-MS, 1994.

[4] P.L. Kerr, et al., "Real-time alpha emitter assay of large volumes," Proc. Waste Management '97, Folio Infobase CD-ROM.

[5] Filtrete is manufactured by $3 \mathrm{M}$ Air Quality Products, 3M Center 223-4S-01, St. Paul, MN 55101.

[6] R.D. Bolton, "Radon monitoring using long-range alpha detector-based technology," Proc. IEEE Nuclear Science Symposium, Norfolk, VA, 1994. 


\section{M98003301}

Report Number (14)LA-UR- $-97-4608$

CONF-971147--

Publ. Date (11)

Sponsor Code (18)

UC Category (19)

$\frac{\frac{199802}{20 E / M A, X F}}{U C-907, D O E / E R}$

